\title{
Trauma and Reiter's syndrome: development of 'reactive arthropathy' in two patients following musculoskeletal injury
}

\author{
JEFFREY J. W IS N I E S K I \\ From the Medical Service, Rheumatology Section, Cleveland Veterans Administration Medical Center, and \\ Case Western Reserve University School of Medicine, Cleveland, Ohio, USA.
}

SUMMARY Two patients are reported who developed arthropathies with some features of Reiter's syndrome shortly after physical injury. Both were HLA-B27 positive. No other precipitating factors were identified, and the possibility that trauma may have precipitated a reactive arthropathy is discussed.

Key words: endogenous antigen (immunogen), genetic predisposition, collagen.

Concepts of Reiter's syndrome (RS) have changed dramatically in the past decade. Notable developments include: (1) recognition that clinical activity may be acute/self-limited, acute/recurrent, or chronic $^{1-5}$; (2) recognition that disease manifestations vary from 'incomplete' RS to multisystem involvement ${ }^{1-3}$; (3) development of concepts of genetic predisposition and linkage ${ }^{4}$; and (4) characterisation of $\mathrm{RS}$ as reactive arthritis developing in genetically predisposed individuals. ${ }^{4}$ In most patients the inciting event is genitourinary or enteric infection. ${ }^{3}$ The following cases suggest that trauma might also provoke a reactive rheumatic syndrome consistent with RS.

\section{Case reports}

\section{CASE 1}

A 23-year-old white man presented with chronic pain, swelling, and stiffness in his right knee. Seven months previously he had struck his knee while rock climbing. Moderate pain and swelling occurred but there was no ecchymosis, locking, or instability. Three days after the injury symptoms and signs had almost resolved; however, on the seventh day pain, swelling, and stiffness recurred. Initial medical evaluation one month after the injury confirmed

Accepted for publication 5 July 1984.

Correspondence to J. J. Wisnieski, MD, Cleveland VA Medical Center, 10701 East Boulevard, Cleveland, Ohio 44106, USA. swelling, pain, and heat; internal mechanical derangement was suspected. Five months after the trauma arthroscopy revealed hyperaemic and thickened synovium but no structural abnormality. Synovial biopsy showed chronic synovitis with marked lymphocyte and plasma cell infiltration.

Seven months after the injury the knee continued to be warm and painful, with thickened synovium and a large effusion. No other joints were involved. Conjunctivitis of the left eye was noted. There was no history of venereal disease, diarrhoea, rash, mucocutaneous ulceration, dysuria, or back pain. 50 $\mathrm{ml}$ of moderately inflammatory (grade II) fluid was removed from the right knee. Serum and joint fluid were negative for rheumatoid factor. Lumbar spine and sacroiliac $x$-rays were normal; knee $x$-rays showed capsular distension and thickening. The blood count was normal and the ESR (Wintrobe) was $22 \mathrm{~mm} / \mathrm{h}$. HLA-B27 was positive.

The patient was treated with rest, indomethacin, and one injection of intra-articular corticosteroid. One month later he was much improved; conjunctivitis had resolved. One year after the trauma the knee was normal, with full range of motion.

CASE 2

A 55-year-old white man was admitted for diagnosis of a recurrent rash, fever, and arthritis. He had been well as an adolescent and young adult. At age 20 he suffered multiple severe contusions in a motor-car 


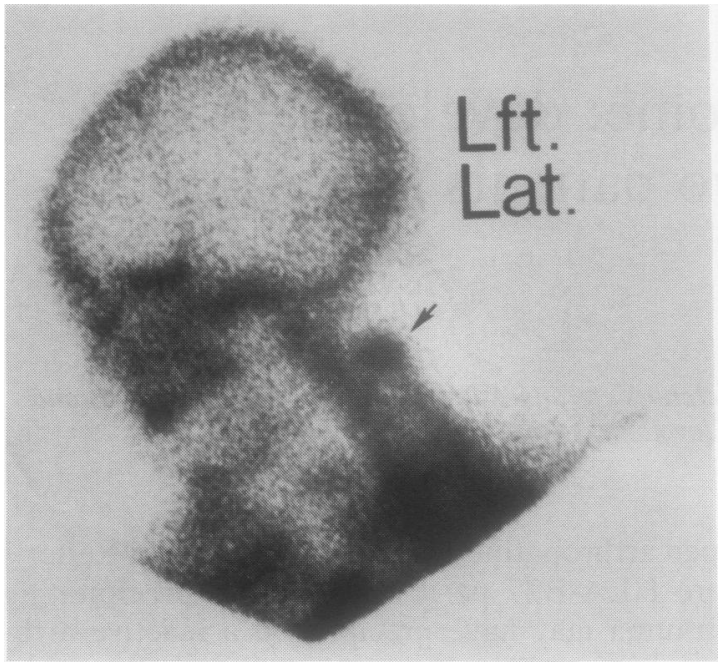

Fig. 1 Ligament involvement in Reiter's syndrome. Note increased uptake of ${ }^{98 \mathrm{~m}}$ technetium MDP in the cervical posterior longitudinal spinous ligament. Uptake is particularly heavy at the ligament insertion on the posterior spine of $C 2$ (arrow).

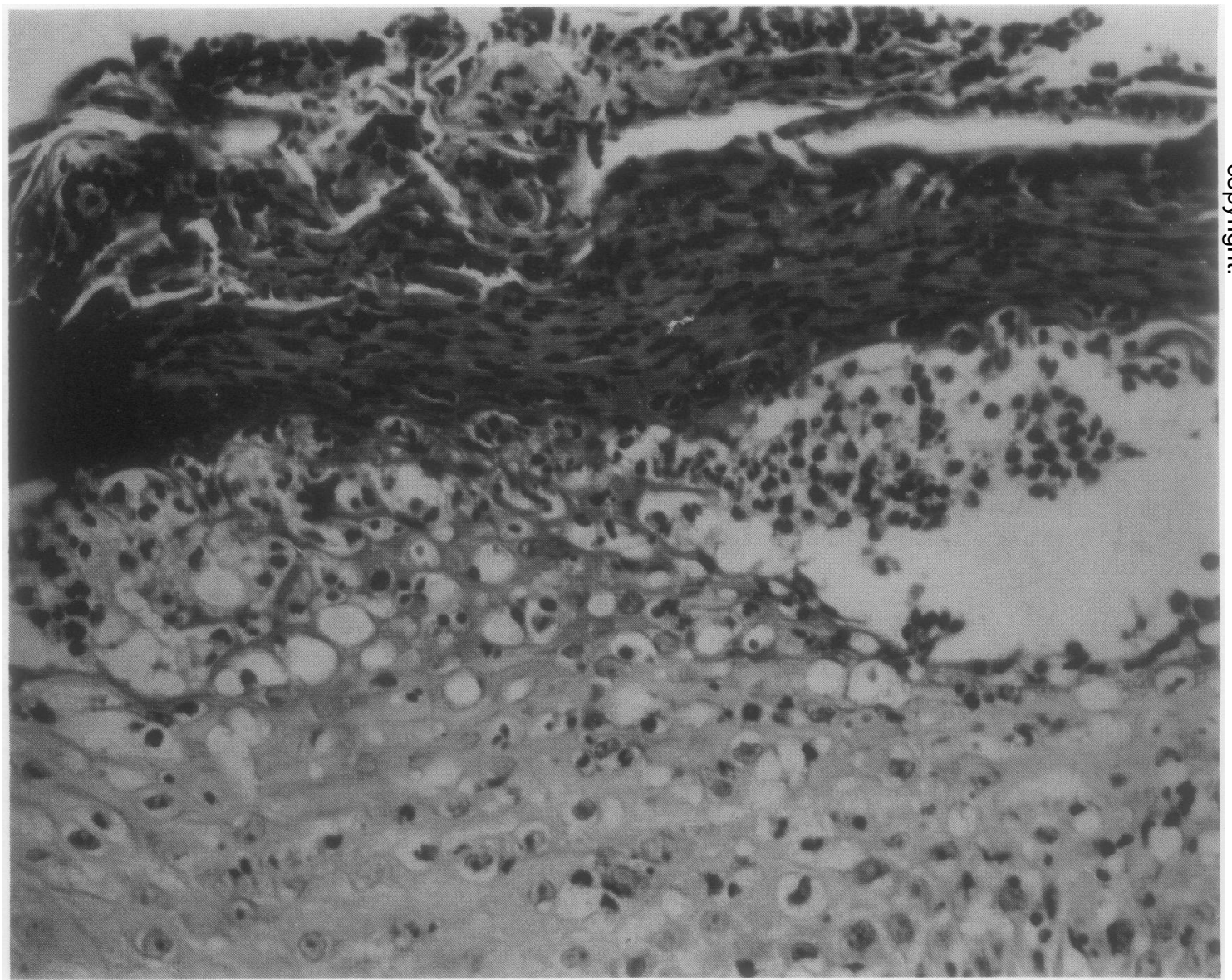

Fig. 2 Keratodermia blennorrhagica. Note acanthosis, hyperkeratosis, and parakeratosis. Degeneration of epidermal cells has occurred, causing a spongiform appearance. Coalescence of these ghost cells and influx of polymorphonuclear leucocytes results in pustule formation. (Haematoxylin and eosin, $\times 80$ ). 
accident. By one month of convalescence his traumatic injuries had almost healed; however, fever, low back pain, tender and swollen joints, and a rash developed three weeks after the trauma. The affected joints were the knees, wrists, and ankles. The rash was scaly and pustular and involved only the extremities.

Episodes of fever, arthritis, rash, and/or low back pain lasting up to 2 to 10 months recurred at irregular intervals throughout his adult life. Mild conjunctivitis and painless mouth ulcers were noted during several recurrences. There was no history of dysuria, gonorrhoea, penile ulcerations, or diarrhoeal illness.

On admission, low grade fever and arthritis of knees, wrists, shoulders and metacarpophalangeal joints were noted. Neck motion was limited and painful. The sacroiliac joints were tender to gentle pressure. There was warmth, swelling, and tenderness on palpation of the posterior ligamentous insertions on the dorsal spines of $\mathrm{C} 2$ and $\mathrm{C} 7$. Mild bilateral conjunctivitis was observed. A few scaly skin lesions $0.5-1.0 \mathrm{~cm}$ in diameter were found on the extremities. The haematocrit was 34 and ESR (Wintrobe) was $55 \mathrm{~mm} / \mathrm{h}$. Joint fluid was moderately inflammatory (grade II). Both serum and joint fluid were negative for rheumatoid factor (RF). A bone scan with technetium methylene diphosphonate showed marked uptake in the sacroiliac, shoulder, knee, ankle, and wrist joints. There was also heavy uptake around the ligament insertion on the spine of C2 (Fig. 1). $X$-rays showed asymmetrical sacroiliitis and non-marginal syndesmophytes of the lumbar spine. Biopsy of a skin lesion was consistent with keratodermia blennorrhagica (Fig. 2). HLA-B27 was positive.

The patient was treated with physiotherapy, indomethacin, and low-dose prednisone. The fever and rash promptly resolved, and the arthritis improved sufficiently to allow self care and ambulation with a walking frame.

\section{Discussion}

The temporal relationship between physical trauma and development of a rheumatic syndrome in these two patients is striking. In case 1 the time between trauma and initial symptoms was seven days, and in case 2 it was 20-25 days. The first patient's illness was consistent with incomplete Reiter's syndrome and was self limited, lasting approximately one year. The second patient had arthritis, conjunctivitis, mouth ulcers, and a rash. The rash never affected his palms or soles. The inflammatory lesion of the posterior spinous ligament on bone scan demonstrates the enthesopathic nature of his illness. He continues symptomatic and disabled 35 years after disease onset.
That trauma initiated a 'Reiter's-like' syndrome in the two patients described above may be a fortuitous observation. None of the current rheumatology texts $^{36-8}$ or reviews of the subject ${ }^{159}$ list trauma as a causative factor. However, psoriatic arthritis, which may closely resemble RS, has been reported as a consequence of musculoskeletal injury in some patients with psoriatic skin disease..$^{11}$ In several patients with only cutaneous psoriasis, a chronic inflammatory arthritis of the knee developed after the knee was injured. ${ }^{11}$ Arthrotomy and synovial biopsy revealed only synovial proliferation consistent with psoriatic arthritis. Similarly, Williams and Scott described a psoriatic patient whose chronic erosive arthritis began shortly after trauma to the right middle finger. Only joints in that finger became arthritic, with periosteal elevation along the proximal phalanx. ${ }^{12}$

How trauma might cause a reactive syndrome with RS features is not clear. Trauma may possibly alter and/or release antigen(s) from connective tissue. Like some Klebsiella antigens, ${ }^{13-15}$ these may interact with B27 or immune response gene products on lymphocyte membranes to influence critically cell viability and function. Through such interaction both bacterial antigens and self-antigens like collagen may trigger cell and antibody mediated responses against host connective tissue, especially synovium and cartilage. In normal persons foreign or self-antigen would not interact with gene/gene products. However, in predisposed individuals interaction may not only occur but result in perpetuation of immune responses which cause clinical disease. Although antibodies against and lymphocyte hyperreactivity to collagen, particularly type II, have been found consistently in rheumatoid arthritis, too few RS patients have been studied to permit conclusions about the incidence or role of these abnormalities in Reiter's syndrome. . $^{16-19}$

Recent studies with rat models indicate a possible role for self-antigens in the pathogenesis of rheumatic disease. Pearson and Chang produced a disease much like RS in inbred rats using either complete Freund's adjuvant or a non-immunogenic synthetic adjuvant. They postulated that the injection and/or adjuvant itself releases potentially immunogenic self-antigen(s). Rheumatic disease may then occur as the result of an abnormal, genetically determined immune response to such antigen(s), facilitated by the adjuvant. ${ }^{20}$ Although the nature of such antigen(s) remains speculative, type II collagen may be an important source of endogenous antigen. Trentham et al. have demonstrated the antigenicity and arthritogenic potential of type II collagen: outbred rats injected intradermally with homologous type II collagen in incomplete Freund's adjuvant developed a 
chronic erosive polyarthritis similar to human rheumatoid arthritis. ${ }^{2122}$ Such studies suggest that response to self-antigen may occur in provocative circumstances. The injection of susceptible animals with complete adjuvant alone or incomplete adjuvant plus homologous antigen may be a 'trauma' analogous to physical injury in man. The former may introduce and/or promote a response to selfantigen(s), while the latter may physically alter and release self-antigen(s) from endogenous connective tissue. In both instances the genetic background may dictate immune response(s) which lead to a reactive rheumatic disease.

\section{References}

1 Good A E. Reiter's disease. A review with special attention to cardiovascular and neurologic sequelae. Semin Arthritis Rheum 1974; 3: 253-86.

2 Arnett F C, McClusky D E, Schacter B Z, Lordon R E. Incomplete Reiter's syndrome: discriminating features and HLA W27 in diagnosis. Ann Intern Med 1976; 84: 8-12.

3 Calin A. Reiter's syndrome. In: Kelley W M, Harris E D, Ruddy S, Sledge C D, eds. Textbook of rheumatology. Philadelphia: Saunders, 1981: 1033-46.

4 Calin A, Fries J F. An 'experimental' epidemic of Reiter's syndrome revisited. Follow-up evidence on genetic and environmental factors. Ann Intern Med 1976; 84: 564-6.

5 Fox R, Calin A, Gerbo R C, Gibson D. The chronicity of symptoms and disability in Reiter's syndrome: an analysis of 131 consecutive patients. Ann Intern Med 1979; 91: 190-3.

6 Sharp J T. Reiter's syndrome. In: McCarty D J ed. Arthritis and allied conditions. 9th ed. Philadelphia: Lea and Febiger, 1979: 633-61.

7 Wright V. Reiter's disease. In: Scott J T, ed. Copeman's textbook of the rheumatic diseases. 5th ed. Edinburgh: Churchill Livingstone, 1978: 549-66.

8 Katz W A. Reiter's disease. In: Katz W A, ed. Rheumatic diseases: diagnosis and management. Philadelphia: Lippincott, 1977: 547-55.

9 Bluestone, R, Pearson C M. Ankylosing spondylitis and Reiter's syndrome: Their interrelationship and association with HLA B27. In: Stollerman G H, Harrington W J, Kirsner J B, Kossman C E, Siperstein M D, eds. Advances in internal medicine. Chicago: Yearbook Medical Publishers, 1978; 22: 1-19.

10 Moll J M H, Wright V. Psoriatic arthritis. Semin Arthritis Rheum 1973; 3: 55-78.

11 Wright V. Psoriatic arthritis. In: Scott J T, ed. Copeman's textbook of rheumatic diseases. 5th ed. Edinburgh: ChurchillLivingstone, 1978; 537-48.

12 Williams K A, Scott J T. Influence of trauma on the development of chronic inflammatory arthritis. Ann Rheum Dis 1967; 26: 532-7.

13 Seager K, Bashir H V, Geczy A R, Edmonds J P, DevereTyndall A. Evidence for a specific B27-associated cell surface marker on lymphocytes of patients with ankylosing spondylitis. Nature 1979; 277: 68-70.

14 Geczy A F, Alexander K, Bashir H V, Edmonds J P. Characterization of a factor(s) present in Klebsiella culture filtrates that specifically modifies an HLA-B27 associated cell-surface component. J Exp Med 1980; 152 (suppl): 331s-40s.

15 Edmonds J, Macauley D, Tyndell A, et al. Lymphocytotoxicity of anti-Klebsiella antiserum in ankylosing spondylitis and related arthropathies. Arthritis Rheum 1981; 24: 1-7.

16 Mestecky J, Miller E J. Presence of antibodies specific to cartilage-type collagen in rheumatoid synovial tissue. Clin Exp Immunol 1975; 22: 453-6.

17 Andriopoulos N A, Mestecky J, Miller E J, Bradley E L. Antibodies to native and denatured collagen in sera of patients with rhe umatoid arthritis. Arthritis Rheum 1976; 19: 613-7.

18 Trentham D E, Dynesius R A, Rocklin R E, David J R. Cellular sensitivity to collagen in rheumatoid arthritis. $N$ Engl J med 1978; 299: 327-32.

19 Smolen J S, Menzel E J, Scherak O, et al. Lymphocyte transformation to denatured type I collagen and B lymphocyte alloantigens in rheumatoid arthritis. Arthritis Rheum 1980; 23: 424-32.

20 Pearson $\mathrm{C} \mathrm{M}$, Chang Y H. Adjuvant disease: pathology and immune reactivity. Ann Rheum Dis 1979; 38 (suppl 1): 102-10.

21 Trentham D E, Townes A S, Kang A H. Autoimmunity to type II collagen: an experimental model of arthritis.J Exp Med 1977; 146: $857-68$.

22 Trentham D E, Townes A S, Kang A H. Humoral and cellular sensitivity to collagen in type II collagen-induced arthritis in rats. J Clin Invest 1979; 61: 89-96. 